\title{
The Inhibitory Effects of Gossypol on Human Sperm Motility Characteristics: Possible Modes of Reversibility of Those Effects
}

\author{
Panayiotis M. Zavos ${ }^{1,2}$ and \\ Panayota N. Zarmakoupis-Zavos ${ }^{1,2}$ \\ ${ }^{1}$ Andrology Institute of Lexington, Lexington, Kentucky \\ 40546, and ${ }^{2}$ Kentucky Center for Reproductive Medicine, \\ Lexington, Kentucky 40508, USA
}

Zavos, P.M. and Zarmakoupis-Zavos, P.N. The Inhibitory Effects of Gossypol on Human Sperm Motility Characteristics: Possible Modes of Reversibility of Those Effects. Tohoku J. Exp. Med., 1996, 179 (3), 167-175 — Gossypol (GOS) is a polyphenolic compound derived mainly from cottonseed oil, which has been found to have anti-fertility effects in males. It has been reported to induce disturbances of the hypothalamicpituitary axis, disruption of spermatogenesis in the testes, and inhibition of postejaculatory spermatozoa motility. The inhibition of human sperm motility by GOS has been documented both in vivo and in vitro, although the exact mechanism and possible reversibility of such inhibition is unknown. The objectives of the present study were 1) to examine the in vitro dose response of human sperm motility to GOS, and 2) to determine if the motility inhibition of GOS was reversible, using agents which alter the second messenger cyclic adenosine monophosphate (cAMP), such as, 8-bromo-cAMP (8-Br-cAMP), and forskolin, and adenylate cyclase stimulator. Fresh spermatozoa were obtained from males of known fertility. Motile spermatozoa were recovered via the SpermPrep ${ }^{\mathrm{TM}}$ (SP) method and used further in all experiments. Quantitative and qualitative sperm parameters were recorded at collection, post-SP filtration and post-treatment. Motile spermatozoa were resuspended in either media (SP), or in increasing concentrations of GOS $(10,20,30$ and $50 \mu \mathrm{g} / \mathrm{ml})$ as gossypol acetic acid in media. To study the possible reversibility of the GOS effects, spematozoa already exposed to GOS for $2 \mathrm{hr}$ (at the concentrations mentioned above) were centrifuged and reconstituted in media containing either $10 \mathrm{mM}$ 8-Br-cAMP or 100 $\mu \mathrm{M}$ forskolin and measurements of percent motility and grade of motility $(0-4)$ were taken at 0 time and at $30 \mathrm{~min}$ intervals for a total of $2 \mathrm{hr}$. Each experiment was replicated 8 times. The results obtained in this study showed that GOS inhibited sperm motility in a dose and time dependent manner. The motility characteristics of the $50 \mu \mathrm{g} / \mathrm{ml}$ GOS group were lower than all other groups ( $p<$ 0.001 ) and the spermatozoa were completely immobilized within 60 min. Cyclic AMP somewhat rescued the GOS-treated sperm, whereas exposure of GOS-treated

Received January 22, 1996; revision accepted for publication April 25, 1996.

Address for reprints: Professor Panayiotis M. Zavos, Ed.S., Ph.D., 607 W.P. Garrigus Bldg., University of KY Lexington, KY 40546, USA.

Presented at the 11th Annual Meeting of the European Society of Human Reproduction and Embryology, Hamburg, Germany, June 28-July 1, 1995. 
sperm to forskolin had no such effects. The data generated in the present study suggest that GOS inhibits cAMP formation, which subsequently decreases sperm motility characteristics. At low concentrations (up to $20 \mu \mathrm{g} / \mathrm{ml}$ for $30 \mathrm{~min}$ ), GOS inhibition is reversible and compounds that act to increase cAMP seem to be partially responsible for the reversal of GOS inhibition. However, GOS inhibitory effects at levels higher than $20 \mu \mathrm{g} / \mathrm{ml}$ (exposed for $30 \mathrm{~min}$ ) were impossible to reverse, which suggest that GOS at those levels could be an effective agent for vaginal contraception.___ gossypol; human spermatozoa; sperm motility

Gossypol is a yellowish polyphenolic compound found in the pigment glands of the seed, leaf, stem and root of the cotton plant, genus Gossypium of the family Malvaceac (Abou-Donia 1970, 1974, 1976). It was initially noted from its toxicological effects on non-ruminant animals when the livestock were fed with cottonseed meal as food supplement. There was renewed interest in gossypol human physiology during 1956-1959, primarily because of the potential for using the high-protein cottonseed flour, Incaparina, as a protein supplement for malnourished children and pregnant women in developing countries. Results indicated that small amounts of gossypol in food was safe for human consumption (Bressani 1980). The elixir extracted from cotton root bark which contains a high concentration of gossypol has long been used traditionally in some countries as an abortifacient and menses inducer (Slocumb 1980). Antitumor activity of gossypol on some ascites carcinoma and solid tumors was also cited (Vermel and Jone 1963). Much of the information indicated that the utilization of gossypol by humans around the world has a long history.

In the 1960s, Chinese workers discovered through mass investigation in some districts in China that cooking with crude cottonseed oil led to infertility in human males. Large-scale animal experiments carried out in 1970s showed that the active ingredient associated with cottonseed oil which induced infertility was gossypol. Following an extensive series of studies using purified gossypol, gossypol-acetic acid and gossypol-formic acid on the antifertility effect, site and mechanism of action, pharmacokinetics, metabolism and toxicity in several species of animals, clinical studies of gossypol as a male contraceptive agent were suggested (Wu 1972; Wang and Lei 1979; Tang 1980; Xue 1981). The first clinical trial of gossypol was started in 1972, and the above three types of gossypol tablet were tested in 14 districts of China (Wu 1972). Over 10,000 men used the drugs for a period ranging from 6 months to 8 years, the overall efficacy is $99.07 \%$ as estimated by sperm examination. Besides an incidence of $0.75 \%$ hypokalemic paralysis which may be related to dietary intake of potassium, no serious toxic side-effects were observed provided the dosage was kept at the antifertility level. The discovery of gossypol's antifertility effect has aroused worldwide attention and interest among andrologists and in the field of population and family planning, because it is a new non-steroid drug which has been tested on more than 10,000 subjects and for far longer periods than has any other agent. 
Today gossypol is regarded as a compound that can cause a variety of effects on a variety of target organs and tissues. Disturbances of the hypothalamicpituitary axis, disruption of spermatogenesis in the testes, and inhibition of postejaculatory spermatozoa motility have been reported. The inhibition of human sperm motility by gossypol has been documented both in vivo and vitro, although the exact mechanism and reversibility is unkhown (Lee and Malling 1981; Chongthammakun et al. 1986). The objectives of the present study were to: 1) examine the in-vitro dose response of human sperm motility to gossypol and 2) determine if the motility inhibition of gossypol was reversible using agents that alter the second messenger cyclic adenosine monophosphate.

\section{Materials and Methods}

\section{Semen specimens}

Fresh human semen samples were obtained from patients that were undergoing infertility workup at the Andrology Institute of Lexington. Patients collected their ejaculates via a Male Factor Pak (ZBL, Inc., Lexington, KY, USA; Zavos 1985; Zavos and Goodpastnre 1989). Specimens were pooled, when necessary, to obtain a concentration of $>50$ million motile sperm per ml. Measurements of sperm parameters were obtained manually using WHO methods and standards. SpermPrep ${ }^{\mathrm{TM}}$ (SP) media (ZBL, Inc.) was used in all wash and SP filtration procedures, and for dilutions of all stock solutions. All specimes were initially washed via centrifugation for $10 \mathrm{~min}$ at $450 \times \mathrm{g}$, and the pellet was then reconstituted and filtered via the SP method (Zavos 1992; Sofikitis et al. 1993). The filtrate from the SP filtration was divided into equal aliquots and centrifuged to obtain pellets with equal numbers of sperm at the beginning of each experiment.

All chemicals used were obtained from the Sigma Chemical Co. (St. Louis, MO, USA). The gossypol solutions were made by dissolving gossypol acetic acid in ether and absolute alcohol $(1: 4)$, resulting in a $20 \mathrm{mg} / \mathrm{ml}$ solution. This was diluted with SP media to yield solutions containing $10,20,30$ and $50 \mu \mathrm{g} / \mathrm{ml}$ of gossypol.

The 8-bromo cyclic adenosine monophospate (8-Br-cAMP) stock solution was made by dissolving in distilled water to a concentration of $100 \mathrm{mM}$, then further diluting with media to $10 \mathrm{mM}$. The forskolin solutions were made by dissolving forskolin in absolute alcohol to a concentration of $10 \mathrm{mM}$, then further diluting with media to $100 \mu \mathrm{M}$.

\section{Experiment 1: Gossypol dose response}

In this experiment, the sperm pellets obtained from the SP filtration procedure were resuspended in either media (control) or varying concentrations $(10,20$, 30 , or $50 \mu \mathrm{g} / \mathrm{ml}$ ) of gossypol acetic acid in media. Manual determinations of motility and count were taken immediately upon initial resuspension of the sperm 
pellets (0 time), and at 30-min intervals thereafter for $2 \mathrm{hr}$, to obtain a gossypol dose-response time course. Eight replicates were performed.

\section{Experiment 2: Gossypol/cyclic AMP interactions}

Sperm obtained from the filtration $\left(\right.$ SpermPrep $^{\mathrm{TM}}$ II) procedure were centrifuged and then resuspended in media (groups 1 and 2), or $20 \mu \mathrm{g} / \mathrm{ml}$ gossypol solution, chosen from the dose-response study as described above in Experiment 1 (groups 3 and 4). Count and motility measurements were taken at the initiation of gossypol treatment and following the 2 -hr incubation period. After centrifugation to remove the sperm from the incubation media, group 1 was resuspended in media (media/media group), group 2, in $10 \mathrm{mM}$ 8-Br-cAMP (media/cAMP group), group 3, in media (gossypol/media group), and group 4 was, in $10 \mathrm{mM} 8$-Br-cAMP (gossypol/cAMP group). Count and motility measurements were again taken at the time of resuspension ( 0 time), and at $30 \mathrm{~min}$ intervals for $2 \mathrm{hr}$. The experiment was replicated 8 times.

\section{Experiment 3: Gossypol/forskolin}

Sperm from the filtration procedure were centrifuged and were then resuspended in media (groups 1 and 2), or in $20 \mu \mathrm{g} / \mathrm{ml}$ gossypol solution (groups 3 and 4). Count and motility measurements were taken at the time of initial resuspension, and following a 2 -hr incubation period. After another centrifugation to remove the sperm from the incubation media, group 1 was resuspended in media (media/media group), group 2, in $100 \mu \mathrm{M}$ forskolin (media/forskolin group), group 3, in media (gossypol/media group), and group 4 , in $100 \mu \mathrm{M}$ forskolin (gossypol/forskolin group). Count and motility measurements were again taken at the time of resuspension ( 0 time), and at 30,60, and $120 \mathrm{~min}$. The experiment was replicated eight times.

\section{Statistical analysis}

Analysis of variance with repeated measurements was performed using SAS program. All measurements are shown as means \pm s.D.

\section{RESULTS}

\section{Experiment 1: Gossypol dose-response}

In this study (Table 1$)$, sperm motility was measured at 30 min intervals $(0$ to $2 \mathrm{hr}$ ) and over a range of gossypol concentrations $(0,10,20,30$, and $50 \mu \mathrm{g} / \mathrm{ml})$. At initial resuspension ( 0 time), the motility of the $50 \mu \mathrm{g} / \mathrm{ml}$ group was lower than all other groups $(p<0.001)$, and was zero by one hour. Motility in the $30 \mu \mathrm{g} / \mathrm{ml}$ group was significantly less than in the 0,10 , and 20 groups $(p<0.05)$ at all times measured except for the zero time. The $20 \mu \mathrm{g} / \mathrm{ml}$ group was significantly different from control only at the 90 -min time point, but was greater than the 30 $\mu \mathrm{g} / \mathrm{ml}$ group at the 30 -min time and beyond $(2 \mathrm{hr}$ ). The $10 \mu \mathrm{g} / \mathrm{ml}$ group was not 
TABLE 1. Effects of various concentrations of gossypol on sperm motility: Dose response measurements

\begin{tabular}{cccccc}
\hline $\begin{array}{c}\text { Gossypol } \\
\text { concentrations } \\
(\mu \mathrm{g} / \mathrm{ml})\end{array}$ & 0 & 30 & 60 & 90 & 120 \\
\cline { 2 - 6 } & $75.3 \pm 6.1^{\mathrm{a}}$ & $73.2 \pm 5.5^{\mathrm{a}}$ & $75.1 \pm 6.0^{\mathrm{a}}$ & $72.3 \pm 5.2^{\mathrm{a}}$ & $71.1 \pm 4.8^{\mathrm{a}}$ \\
Control & $74.1 \pm 5.3^{\mathrm{a}}$ & $72.2 \pm 5.8^{\mathrm{a}}$ & $70.1 \pm 6.1^{\mathrm{a}}$ & $71.2 \pm 5.0^{\mathrm{a}}$ & $69.3 \pm 4.9^{\mathrm{a}}$ \\
10 & $70.1 \pm 6.3^{\mathrm{a}}$ & $63.3 \pm 6.0^{\mathrm{a}}$ & $60.1 \pm 5.5^{\mathrm{a}}$ & $57.1 \pm 4.9^{\mathrm{b}}$ & $55.8 \pm 5.2^{\mathrm{b}}$ \\
20 & $68.2 \pm 7.1^{\mathrm{a}}$ & $40.3 \pm 5.8^{\mathrm{b}}$ & $35.5 \pm 4.2^{\mathrm{b}}$ & $32.1 \pm 3.8^{\mathrm{c}}$ & $25.6 \pm 4.1^{\mathrm{c}}$ \\
30 & $39.9 \pm 8.2^{\mathrm{b}}$ & $15.1 \pm 3.5^{\mathrm{c}}$ & 0.0 & 0.0 & 0.0 \\
50 & & & & & \\
\hline
\end{tabular}

${ }^{a-c}$ Means followed by same letter are not different per time period $(p>$

0.05). Eight replications were performed.

(Means \pm s.E.)

different from control at any time measured.

Experiment 2: Gossypol/cyclic AMP interactions

Gossypol treatment $(20 \mu \mathrm{g} / \mathrm{ml})$ for the 2 -hr incubation period reduced motility in the two gossypol-treated groups compared with the two media-treated groups $(60 \%$ and $62 \%$ vs. $81 \%$ and $80 \%$, respectively, $p<0.001$; Table 2$)$. After $30 \mathrm{~min}$ of cAMP treatment, the gossypol/media sperm had lower motility compared to the media/cAMP, media/media, and gossypol/cAMP groups $(p<0.05)$. Cyclic AMP rescued the gossypol-treated sperm for the first $30 \mathrm{~min}$ of treatment, so that motility in the gossypol/cAMP group was not significantly less than the media/media control group. The motility of the gossypol/cAMP group declined in parallel with the gossypol/media group over the next $90 \mathrm{~min}$. At $120 \mathrm{~min}$, all four groups had significantly different motilities. The motility of control (media/cAMP) sperm was enhanced in a similar manner.

TABLE 2. The effects of c-AMP on the gossypol inhibition: Sperm motility measurements over time of incubation

\begin{tabular}{|c|c|c|c|c|c|c|c|c|}
\hline & \multirow{2}{*}{ Treatment groups } & \multicolumn{7}{|c|}{ Time intervals (min) } \\
\hline & & $0^{1}$ & $120^{1}$ & $0^{1}$ & 30 & 60 & 90 & 120 \\
\hline 1 & Control & $82.1^{\mathrm{a}}$ & $81.6^{\mathrm{a}}$ & $82.3^{\mathrm{a}}$ & $80.7^{\mathrm{a}}$ & $81.8^{\mathrm{a}}$ & $80.3^{\mathrm{a}}$ & $79.6^{\mathrm{a}}$ \\
\hline 2 & c-AMP & $80.3^{\mathrm{a}}$ & $80.2^{\mathrm{a}}$ & $81.6^{\mathrm{a}}$ & $82.6^{\mathrm{a}}$ & $83.7^{\mathrm{a}}$ & $85.1^{\mathrm{a}}$ & $87.2^{\mathrm{b}}$ \\
\hline 3 & Gossypol & $79.8^{\mathrm{a}}$ & $60.2^{\mathrm{b}}$ & $61.3^{\mathrm{b}}$ & $50.7^{\mathrm{b}}$ & $45.3^{\mathrm{b}}$ & $41.2^{\mathrm{b}}$ & $33.4^{c}$ \\
\hline 4 & Gossypol/c-AMP & $80.5^{\mathrm{a}}$ & $62.1^{\mathrm{b}}$ & $61.6^{\mathrm{a}}$ & $57.4^{\mathrm{b}}$ & $54.7^{\mathrm{c}}$ & $50.2^{\mathrm{c}}$ & $46.1^{\mathrm{d}}$ \\
\hline
\end{tabular}

${ }^{a-d}$ Means followed by same letter are not different per time period $(p>0.05)$.

${ }^{1}$ Spermatozoa were incubated with $20 \mu \mathrm{g} / \mathrm{ml}$ of gossypol for $2 \mathrm{hr}$ then washed and incubated in $10 \mathrm{mM}$ c-AMP for 2 additional $\mathrm{hr}$. 
TABLE 3. The effects of forskolin on the gossypol inhibition: Sperm motility measurements over time of incubation

\begin{tabular}{|c|c|c|c|c|c|c|c|c|}
\hline & \multirow{2}{*}{ Treatment groups } & \multicolumn{7}{|c|}{ Time intervals (min) } \\
\hline & & $0^{1}$ & $120^{1}$ & $0^{1}$ & 30 & 60 & 90 & 120 \\
\hline 1 & Control & $80.7^{\mathrm{a}}$ & $81.6^{\mathrm{a}}$ & $80.3^{\mathrm{a}}$ & $81.0^{\mathrm{a}}$ & $82.3^{\mathrm{a}}$ & $81.6^{\mathrm{a}}$ & $80.8^{a}$ \\
\hline 2 & c-AMP & $81.2^{\mathrm{a}}$ & $83.4^{\mathrm{a}}$ & $84.2^{\mathrm{a}}$ & $85.3^{\mathrm{a}}$ & $86.1^{\mathrm{a}}$ & $86.8^{\mathrm{a}}$ & $87.2^{\mathrm{b}}$ \\
\hline 3 & Gossypol & $81.8^{\mathrm{a}}$ & $76.7^{\mathrm{b}}$ & $72.3^{\mathrm{b}}$ & $68.4^{\mathrm{b}}$ & $62.5^{\mathrm{b}}$ & $54.1^{\mathrm{b}}$ & $45.7^{\circ}$ \\
\hline 4 & Gossypol/c-AMP & $80.9^{\mathrm{a}}$ & $79.5^{\mathrm{b}}$ & $77.8^{\mathrm{b}}$ & $78.4^{\mathrm{b}}$ & $71.8^{\mathrm{c}}$ & $70.2^{c}$ & $68.3^{\circ}$ \\
\hline
\end{tabular}

${ }^{a-d}$ Means followed by same letter are not different per time period $(p>0.05)$.

${ }^{1}$ Spermatozoa were incubated with $20 \mu \mathrm{g} / \mathrm{ml}$ of gossypol for $2 \mathrm{hr}$ then washed and incubated in $100 \mathrm{mM}$ forskolin for 2 additional $\mathrm{hr}$.

\section{Experiment 3: Gossypol/forskolin}

In experiment 3 (Table 3), after $30 \mathrm{~min}$ of exposure to $100 \mu \mathrm{M}$ forskolin, the motility of media/forskolin and gossypol/forskolin groups were not different from each other, but both were higher than the control (media/media) group. This pattern was maintained throughout the remainder of the experiment, although by $120 \mathrm{~min}$, the gossypol/forskolin group was the same as the control group.

\section{Disctession}

In the present studies, we have demonstrated that the anti-motility effects of gossypol on post-ejaculatory human sperm are both dose and time related. At low doses, gossypol does not appear to inhibit motility, but at higher doses, it appears to have an immediate immobilizing effect on a proportion of sperm, with the remainder becoming immotile within a short period of time (30-60 min). This immediate effect of high doses of gossypol $(50 \mu \mathrm{g} / \mathrm{ml})$ may be related to gossypol-induced generation of lipid peroxides and subsequent membrane damage. Other possible mechanisms of action of gossypol include inhibition of lactate dehydrogenase-X and uncoupling of oxidative phosphorylation (Giridharan et al. 1989).

The effects of the lower doses of gossypol $(10 \mu \mathrm{g} / \mathrm{ml})$ over time may be explained by a cAMP-mediated mechanism (Kalla 1981). Gossypol has been shown to reversibly inhibit adenylate cyclase, possibly by competition with ATP at the nucleotide-binding domain of the catalytic subunit of the enzyme (Tso and Lee 1982a). Gossypol has also been shown to inhibit other species sperm adenylate cyclase by chelating manganese, which is a required cofactor (Tso and Lee 1982b).

The reversal of the gossypol effect when 8-Br-cAMP was added, even following a moderately high dose of gossypol $(20 \mu \mathrm{g} / \mathrm{ml})$ for a long incubation period (2 $\mathrm{hr}$ ), is in agreement with previous studies in which gossypol-treated human sperm 
were incubated with dibutyryl-cAMP.

The cAMP-mediated second messenger system has been shown to be involved in the stimulation of sperm motility and metabolism (Kanwar et al. 1990). In human sperm, a positive correlation has been shown between cAMP content, adenylate cyclase activity, and sperm motility. Cyclic AMP has been shown to activate (phosphorylate) an endogenous cAMP-dependent protein kinase, which in turn activates a calcium-controlled motility-regulating protein, resulting in the activation of sperm motility as they are released from storage in the male reproductive tract (Kalla et al. 1986; Brokaw 1987). This is consistent with other in vitro studies while, it conflicts with others, and still others have shown mixed results. The inconsistency in the effect of exogenous cAMP may be due to the use of different analogs of cyclic AMP or to the relatively low permeability of sperm plasma membranes to cyclic nucleotides (Ashizawa et al. 1989; Hammitt et al. 1989).

In the forskolin studies, the inhibition of sperm motility by gossypol at the higher dose used in this investigation $(20 \mu \mathrm{g} / \mathrm{ml})$ was not significantly reversed. This lack of effect at this dose may be due to damage to the sperm plasma membrane by gossypol observed by other investigators.

The present study strongly supports the hypothesis that gossypol inhibits cAMP formation to decrease sperm motility. At low concentrations, gossypol inhibition is reversible, and compounds that act to increase intracellular cAMP at least partially tend to reverse this effect.

The 8-Br-cAMP reversal of the anti-motility effect of gossypol is consistent with gossypol acting at the level of cAMP formation or metabolism, upstream from its cAMP-dependent protein kinase. The stimulator of adenylate cyclase, forskolin, reverse the inhibitory effects of gossypol on sperm motility confirming a cAMP-mediated mechanism of action. It is possible that gossypol is acting both to prevent the formation of $\mathrm{cAMP}$ by adenylate cyclase and to stimulate the metabolism by phosphodiesterase.

It appears that gossypol also acts rapidly making its effect irreversible by agents which regulate cAMP. Therefore, the inhibitory effect of gossypol may make it useful as a vaginal contraceptive. Studies in this area involving animal, as well as, in vitro human subjects, support the possible development of gossypol as a contraceptive agent.

\section{References}

1) Abou-Donia, M.B. (1970) Metabolic fate of gossypol: The metabolism of ${ }^{14} \mathrm{C}$ gossypol in rats. Lipids, $\mathbf{5}, 938$.

2) Abou-Donia, M.B. (1974) Gossypol: Uncoupling of respiratory chain and oxidative phosporylation. Life Sci., 14, 1955-1963.

3) Abou-Donia, M.B. (1976) Physiological effects and metabolism of gossypol. In: Residue Reviews, edited by F.A. Gunther, (Ed.) 61, 126-152.

4) Ashizawa, K., Suzuki, Y. \& Okauchi, K. (1989) Flagellar movement in demem- 
branated preparations of ejaculated fowl spermatozoa. J. Reprod. Fert., 86, 263-270.

5) Bressani, R. (1980) Human nutrition and gossypol. Presented at PARFR Workshop on Gossypol, Program for Applied Research on Fertility Regulation. 11 March, Chicago, Ill.

6) Brokaw, C.J. (1987) Regulation of sperm flagellar motility by calcium and cAMPdependent phosphorylation. J. Cell. Biochem., 35, 175-184.

7) Chongthammakun, S., Ekavipat, C., Sanitwongse, B. \& Pavasuthipaisit, K. (1986) Effects of gossypol on human and monkey sperm motility in vitro. Contraception, 34, 323-331.

8) Giridharan, N., Bamji, M.S. \& Sankaram, A.V.B. (1989) Inhibition in rat testis LDH-X activity by gossypol. Contraception, 26, 607-615.

9) Hammitt, D.G., Bedia, E., Rogers, P.R., Syrop, C.H., Donovan, J.F. \& Williamson, R.A. (1989) Comparison of motility stimulants for cryopreserved human semen. Fertil. Steril., 52, 495-501.

10) Kalla, N.R. (1981) Studies on the male antifertility agent-gossypol acetic acid II. Effect of gossypol acetic acid on the motility and ATPase activity of human spermatozoa. Andrologia, 13, 95-98.

11) Kalla, N.R., Gadru, N. \& Foo, T.W. (1986) Studies on the male antifertility agent gossypol acetic acid VII. Effect of motility stimulated factors on the revival of human spermatozoal motility after gossypol treatment in vitro. Andrologia, 18, 393397.

12) Kanwar, U., Kaur, R., Chadha, S. \& Sanyal, S. (1990) Gossypol-induced inhibition of glucose uptake in human ejaculated spermatozoa may be mediated by lipid peroxidation. Contraception, 42, 573-587.

13) Lee, C.Y. \& Malling, H.Y. (1981) Selective inhibition of sperm specific lactate dehydrogenase $\mathrm{X}$ by an antifertility agent, gossypol. Fed. Proc., 40, 718.

14) Slocumb, J. (1980) Medical and sociological aspects of gossypol use among women in Southwest United States. Presented at PARFR Workshop on Gossypol, Program for Applied Research on Fertility Regulation, 11 March, Chicago, Ill.

15) Sofikitis, N., Miyagawa, I. \& Zavos, P.M. (1993) Selection of single-stranded deoxyribonucleic acid spermatozoa via the SpermPrep filtration column. Fertil. Steril., 59, 690-692.

16) Tang, X.C. (1980) Comparative studies on the absorption, distribution and excretion of ${ }^{14} \mathrm{C}$-gossypol in four species of animals. Acta Pharm. Sin., 15, 212-217.

17) Tso, W.W. \& Lee, C.S. (1982a) Effect of gossypol on boar spermatozoa adenosine triphosphate metabolism. Arch. Androl., 9, 319-331.

18) Tso, W.W. \& Lee, C.S. (1982b) Gossypol: An effective acrosin blocker. Arch. Androl., 8, 143-147.

19) Vermel, E.M. \& Jone, S. (1963) Antitumor activity of gossypol in experiments on transplantable tumors. Voprosy Onkologii, 9, 39. (in Russian)

20) Wang, N.G. \& Lei, H.P. (1979) Antifertility effect of gossypol acetic acid on male rats. Document of 2nd National Conference on Male Antifertility Agens, Qingdao, 1973.

21) Wu, Xi-Rui (1972) Study of the antifertility action of cottonseed and the effective component-gossypol. Document and Presentation at the National Conference on Recent Advance of Family Planning Research, Beijing.

22) Xue, S.P. (1981) Studies on the antifertility effect of gossypol. A new contraceptive for males. Recent Advances in Fertility Regulation, Proceedings of a Symposium, Beijing, September, 1980, 122-146.

23) Zavos, P.M. (1985) Characteristics of human ejaculates collected via masturbation and a new Silastic seminal fluid collection device. Fertil. Steril., 43, 491-492.

24) Zavos, P.M. (1992) Preparation of human frozen-thawed seminal specimens using the SpermPrep filtration method: Improvements over the conventional swim-up 
method. Fertil. Steril., 57, 1326-1330.

25) Zavos, P.M. \& Goodpasture, J.C. (1989) Clinical improvements of specific seminal deficiencies via intercourse with a seminal collection device versus masturbation. Fertil. Steril., 51, 190-193. 